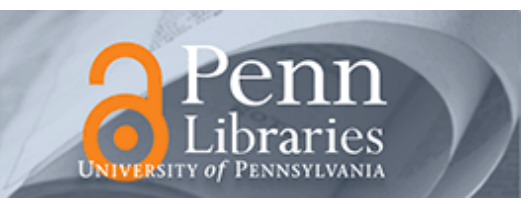

University of Pennsylvania

ScholarlyCommons

$11-6-2012$

\title{
A Model for Emission Yield from Planar Photocathodes Based on Photon-Enhanced Thermionic Emission or Negative-Electron- Affinity Photoemission
}

\author{
Kunal Sahasrabuddhe \\ Stanford University \\ Jared W. Schwede \\ Stanford University \\ Igor Bargatin \\ University of Pennsylvania, bargatin@seas.upenn.edu \\ Joel Jean \\ Massachusetts Institute of Technology

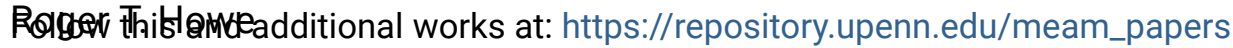 \\ Stanford University \\ Part of the Mechanical Engineering Commons
}

\footnotetext{
See next page for additional authors

Recommended Citation

Sahasrabuddhe, Kunal; Schwede, Jared W.; Bargatin, Igor; Jean, Joel; Howe, Roger T.; Shen, Zhi-Xun; and Melosh, Nicholas A., "A Model for Emission Yield from Planar Photocathodes Based on Photon-Enhanced Thermionic Emission or Negative-Electron-Affinity Photoemission" (2012). Departmental Papers (MEAM). 297.

https://repository.upenn.edu/meam_papers/297
}

Sahasrabuddhe, K., Schwede, J., Bargatin, I., Jean, J., Howe, R., Shen, Z., \& Melosh, N. (2012). A model for emission yield from planar photocathodes based on photon-enhanced thermionic emission or negative-electron-affinity photoemission. Journal of Applied Physics, 112(9), 094907. doi: 10.1063/1.4764106

Copyright 2012 American Institute of Physics. This article may be downloaded for personal use only. Any other use requires prior permission of the author and the American Institute of Physics.

This paper is posted at ScholarlyCommons. https://repository.upenn.edu/meam_papers/297

For more information, please contact repository@pobox.upenn.edu. 


\title{
A Model for Emission Yield from Planar Photocathodes Based on Photon- Enhanced Thermionic Emission or Negative-Electron-Affinity Photoemission
}

\begin{abstract}
A general model is presented for electron emission yield from planar photocathodes that accounts for arbitrary cathode thickness and finite recombination velocities at both front and back surfaces. This treatment is applicable to negative electron affinity emitters as well as positive electron affinity cathodes, which have been predicted to be useful for energy conversion. The emission model is based on a simple one-dimensional steady-state diffusion treatment. The resulting relation for electron yield is used to model emission from thin-film cathodes with material parameters similar to GaAs. Cathode thickness and recombination at the emissive surface are found to strongly affect emission yield from cathodes, yet the magnitude of the effect greatly depends upon the emission mechanism. A predictable optimal film thickness is found from a balance between optical absorption, surface recombination, and emission rate.

\section{Disciplines \\ Mechanical Engineering}

\section{Comments}

Sahasrabuddhe, K., Schwede, J., Bargatin, I., Jean, J., Howe, R., Shen, Z., \& Melosh, N. (2012). A model for emission yield from planar photocathodes based on photon-enhanced thermionic emission or negativeelectron-affinity photoemission. Journal of Applied Physics, 112(9), 094907. doi: 10.1063/1.4764106

Copyright 2012 American Institute of Physics. This article may be downloaded for personal use only. Any other use requires prior permission of the author and the American Institute of Physics.
\end{abstract}

\section{Author(s)}

Kunal Sahasrabuddhe, Jared W. Schwede, Igor Bargatin, Joel Jean, Roger T. Howe, Zhi-Xun Shen, and Nicholas A. Melosh 


\title{
A model for emission yield from planar photocathodes based on photon-enhanced thermionic emission or negative-electron-affinity photoemission
}

\author{
Kunal Sahasrabuddhe, ,,2,a) Jared W. Schwede, ${ }^{1,2,3, a)}$ Igor Bargatin, ${ }^{4,5}$ Joel Jean,, ${ }^{6, b}$ \\ Roger T. Howe, ${ }^{4}$ Zhi-Xun Shen, ${ }^{1,2,3}$ and Nicholas A. Melosh ${ }^{1,3,7, c)}$ \\ ${ }_{1}^{1}$ Geballe Laboratory for Advanced Materials, Stanford University, Stanford, California 94305, USA \\ ${ }^{2}$ Department of Physics and Applied Physics, Stanford University, Stanford, California 94305, USA \\ ${ }^{3}$ Stanford Institute for Materials and Energy Sciences, SLAC National Accelerator Laboratory, \\ 2575 Sand Hill Road, Menlo Park, California 94025, USA \\ ${ }^{4}$ Department of Electrical Engineering, Stanford University, Stanford, California 94305, USA \\ ${ }^{5}$ Department of Mechanical Engineering and Applied Physics, University of Pennsylvania, Philadelphia, \\ Pennsylvania 19104, USA \\ ${ }^{6}$ Department of Electrical Engineering and Computer Science, Massachusetts Institute of Technology, \\ Cambridge, Massachusetts 02139, USA \\ ${ }^{7}$ Department of Materials Science and Engineering, Stanford University, Stanford, California 94305, USA
}

(Received 7 August 2012; accepted 9 October 2012; published online 6 November 2012)

\begin{abstract}
A general model is presented for electron emission yield from planar photocathodes that accounts for arbitrary cathode thickness and finite recombination velocities at both front and back surfaces. This treatment is applicable to negative electron affinity emitters as well as positive electron affinity cathodes, which have been predicted to be useful for energy conversion. The emission model is based on a simple one-dimensional steady-state diffusion treatment. The resulting relation for electron yield is used to model emission from thin-film cathodes with material parameters similar to GaAs. Cathode thickness and recombination at the emissive surface are found to strongly affect emission yield from cathodes, yet the magnitude of the effect greatly depends upon the emission mechanism. A predictable optimal film thickness is found from a balance between optical absorption, surface recombination, and emission rate. (C) 2012 American Institute of Physics. [http://dx.doi.org/10.1063/1.4764106]
\end{abstract}

\section{INTRODUCTION}

Photocathode development has been an active area of research for several decades, driven by applications in photon detectors, electron sources, and photomultipliers. ${ }^{1-8}$ Cathodes with negative electron affinity (NEA), in which the vacuum level is below the conduction band of semiconductor photocathode film, have been especially well studied experimentally due to the high probabilities of electron emission in such films. ${ }^{9-11}$ In these NEA materials, photoexcited electrons impinging upon the surface-vacuum interface have average energies higher than the vacuum level, leading to high electron emission efficiency. However, a new device design has pointed out that electron emission from a material where electrons must first overcome a positive electron affinity energy barrier could be useful for energy conversion. ${ }^{12}$ In this scenario, excited electrons collide with the material surface multiple times on average before escaping, making factors that could be reasonably simplified in NEA materials much more significant, especially surface recombination and electron diffusion. Here, we present an analysis of photoelectron yield that includes a detailed treatment of finite thick-

\footnotetext{
${ }^{a}$ K. Sahasrabuddhe and J. W. Schwede contributed equally to this work.

b) This research was performed while the author was at the Department of Electrical Engineering, Stanford University, Stanford, California 94305, USA.

c) Author to whom correspondence should be addressed. Electronic mail: nmelosh@stanford.edu.
}

ness and surface recombination at both the emissive and back emitter surfaces appropriate for both positive and negative electron affinity materials.

The fundamental photoemission process is typically described in terms of Spicer's three-step model. ${ }^{13}$ Emission is broken down into the following steps: absorption of photons to promote electrons to the semiconductor cathode's conduction band, transport of photoexcited carriers to the surface, and emission or recombination at the cathode's surface. For NEA cathodes, the simplest expression for photoelectron yield is

$$
Y=B \frac{\alpha}{\alpha+L^{-1}},
$$

where $\alpha$ is the absorption coefficient, $L$ the diffusion length of electrons in the conduction band, and $B$ the probability that an electron approaching the surface escapes into vacuum rather than recombining at the surface. This equation has been very fruitful in practice and continues to be used to model NEA photocathodes. ${ }^{14,15}$ However, this relation makes two simplifications: it assumes that the cathode is semi-infinite in extent, and that every electron reaching the surface either escapes or recombines there. Further models have relaxed these assumptions independently, ${ }^{9,16-18}$ yet for most applications it has not been necessary to relax both simplifications simultaneously and examine the interplay between thickness and recombination. 
In contrast, both cathode thickness and surface recombination are expected to have large impacts on emission yield for positive electron affinity (PEA) devices where the average electron energy is below the vacuum level. For example, in a recently described solar energy conversion device based on photon-enhanced thermionic emission (PETE), electrons in the conduction band must rely upon thermal energy to overcome a positive electron affinity at the surface to escape into vacuum. As a result, and in contrast to the high emission probabilities seen in NEA photocathodes, electrons in PEA cathodes relying on PETE have low per-collision probabilities of emission at the surface. Electrons may therefore need to encounter the surface multiple times in order to gain sufficient energy to escape into vacuum, favoring the use of thin films, and requiring an analysis of the emission process that accounts for the effects of both surface recombination and thickness.

In this paper, we present an analytical expression for emission yield under low injection that accounts for the effects of surface recombination, bulk recombination, and cathode thickness. The derivation of this expression is based on a simple steady-state diffusion model that has been successfully used to describe photoluminescence and other semiconductor processes. ${ }^{19,20}$ We then analyze the resulting expression to evaluate the effects of recombination at the emissive front surface, recombination at the non-emissive back surface, and light absorption in a finite film. By examining the theoretical yield for a range of emission velocities in each case, we show how these parameters impact the performance of both NEA cathodes and cathodes based on PETE. Our analysis focuses on cathodes with material properties similar to p-type GaAs, which has been extensively used for photocathode applications and whose bandgap makes it well-suited for use in PETE-based solar energy converters. ${ }^{12}$ Finally, as a practical example, we extend the analysis in the context of PETE applications, including its explicit temperature dependence.

\section{DERIVATION OF EMISSION YIELD}

In this section, we derive an analytical expression for emission yield from a planar semiconductor cathode of thickness $d$ under low injection. The derivation follows the three steps of the Spicer model: photon absorption, photoexcited electron transport, and electron emission. Photon absorption is modeled using a simple exponential profile representing a single pass of incident light through the cathode. Transport is treated using a one-dimensional steady-state diffusion model. Electron emission and surface recombination are incorporated through the boundary conditions of the diffusion model. The result is an analytical expression for emission yield that includes finite thickness and recombination at both surfaces.

When an incident photon excites an electron into a cathode's conduction band, the electron rapidly thermalizes to the cathode's temperature and diffuses throughout the material (Fig. 1(a)). For planar photocathodes, the population of these photoexcited electrons $n$ can be described using a onedimensional diffusion model in steady-state (e.g., Ref. 9),

$$
D \frac{d^{2} n}{d x^{2}}=\frac{n}{\tau}-G(x)
$$

(a)

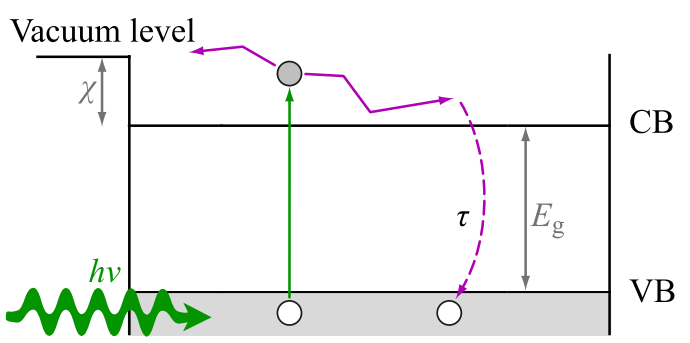

(b)

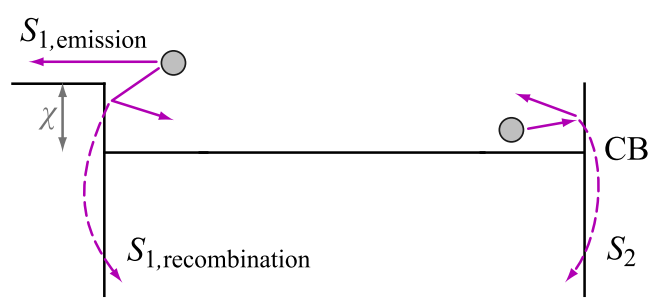

Front (emissive) surface

(surface 1)

$x=0$

Back surface

(surface 2)

$x=d$

FIG. 1. Illustration of the various processes charge carriers undergo in the cathode. (a) Incident photons with energy larger than the bandgap $\left(E_{\mathrm{g}}\right)$ excite electrons from the valence band (VB) to the conduction band (CB). These electrons thermalize and diffuse throughout the cathode; if they do not first recombine in the bulk, as shown with a dashed line, they can reach the surfaces. (b) At the front (emissive) surface (surface 1), electrons may reflect, recombine with surface traps or, if they have sufficient thermal energy, escape into the vacuum. At the back surface (surface 2), they may reflect or recombine. While this figure is drawn with a positive electron affinity $\chi$, such that electrons must overcome an energy barrier to escape at the emissive surface, the equations derived here are also valid for a negative electron affinity (NEA) emitter, where the vacuum level is lowered below the conduction band minimum.

Here, $x$ is the perpendicular distance from the emissive surface, $G(x)$ is the rate of photoexcitation of conductionband electrons, $D$ is the electron diffusion coefficient, and $\tau$ is the bulk recombination lifetime, which, under low injection, is assumed to be independent of the concentration of photoexcited electrons. The cathode is assumed to be free of internal fields, and electrons are assumed to be fully thermalized. It should be noted that a fraction of electrons that are excited by high energy photons can encounter the surface prior to thermalizing to the lattice temperature. This additional contribution to emission current can be treated by considering models for hot-electron photoemission ${ }^{21}$ or by incorporating thermalization into higher energy valleys in the diffusion model used here. ${ }^{9,22}$ Here, we consider only photoexcited electrons, neglecting purely thermal "dark" emission. The current from the small equilibrium concentration of thermally excited electrons can be treated similarly.

Photoexcited electrons that do not recombine in bulk may encounter the front surface, where they can do one of three things: reflect, escape into vacuum, or recombine (Fig. 1(b)). The total current from electrons leaving the conduction band at the front surface (surface 1) is thus,

$$
J_{1}=\left.q_{e} D \frac{d n}{d x}\right|_{x=0}=\left.\left(S_{1, \text { recombination }}+S_{1, \text { emission }}\right) q_{e} n\right|_{x=0} \text {. }
$$

Here, $q_{e}$ is the electron charge, $S_{1, \text { emission }}$ is the emission velocity, a measure of the rate at which electrons escape into vacuum at the emissive surface, and $S_{1 \text {,recombination }}$ is the 


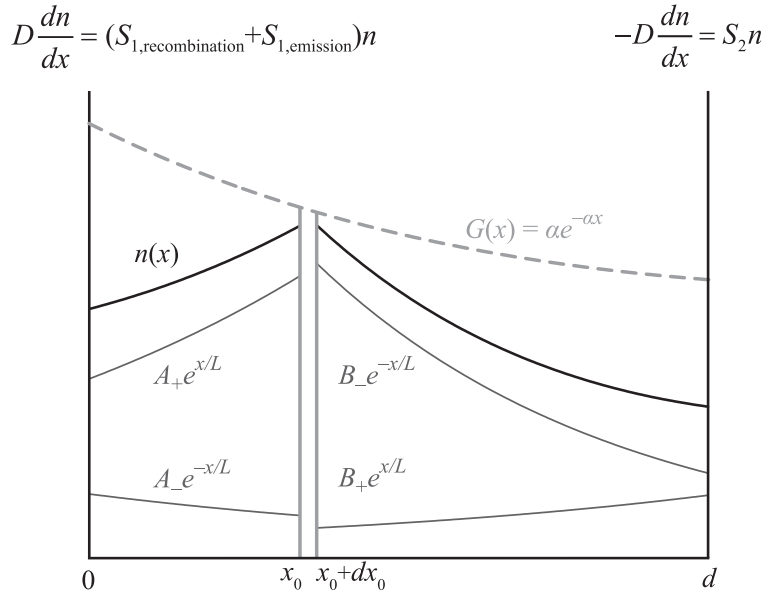

FIG. 2. Exponential solutions to the generation-free diffusion equation apply on either side of a region (from $x_{0}$ to $x_{0}+d x_{0}$ ) in which carrier generation occurs.

surface recombination velocity. For simplicity, we have ignored band-bending at the surface to allow transport in the cathode to be described by the field-free Eq. (2). In practice, band-bending can be incorporated into this model by redefin-

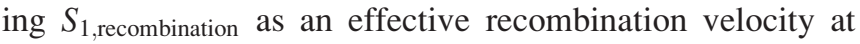
the interface between the low-field bulk and the bandbending region. ${ }^{23-25}$

At the back surface (surface 2 in Fig. 1(b)), we assume that electrons cannot be emitted due to the presence of a substrate, and the recombination current $J_{2}$ is

$$
\frac{J_{2}}{q_{e}}=-\left.D \frac{d n}{d x}\right|_{x=d}=\left.S_{2} n\right|_{x=d},
$$

where $S_{2}$ is the recombination velocity at the back surface.

Assuming generation at a distance $x_{0}$ from the emissive surface results in a general solution $n=A_{+} e^{x / L}+A_{-} e^{-x / L}$ and $n=B_{+} e^{x / L}+B_{-} e^{-x / L}$ on each side of the generation (Fig. 2). The boundary conditions in Eqs. (3) and (4) and continuity of charge carrier density at $x=x_{0}$ allow these coefficients $A_{ \pm}$and $B_{ \pm}$to be related to the recombination velocities at the front and back surfaces. The resulting emission current density $d J_{1, \text { emission }}$ due to generation $G\left(x_{0}\right)$ in a region of thickness $d x_{0}$ is

$$
\frac{d J_{1, \text { emission }}}{q_{e}}=\frac{S_{1, \text { emission }}}{\left(S_{1, \text { emission }}+S_{1, \text { recombination }}\right)\left(1+\zeta_{2} e^{-2 d / L}\right)+D / L\left(1-\zeta_{2} e^{-2 d / L}\right)}\left(e^{-x_{0} / L}+\zeta_{2} e^{-\left(2 d-x_{0}\right) / L}\right) G\left(x_{0}\right) d x_{0} .
$$

Here, $L=\sqrt{D \tau}$ is the electron diffusion length, and we have defined a dimensionless reduced rate of surface recombination at the back surface $\zeta_{2}=\left(D / L-S_{2}\right) /\left(D / L+S_{2}\right)$, which compares recombination at the back surface to recombination in the bulk. The emission yield resulting from generation at $x_{0}$ is found by normalizing the front-surface emission current (Eq. (5)) by the incident photon flux $I_{0}: d Y=d J_{1, \text { emission }} /\left(q_{e} I_{0}\right)$.

The total electron emission yield is found by inserting the relevant generation function and integrating over the device thickness. In a cathode in reflection mode, wherein electron emission occurs from the surface on which light is incident, the generation function is the absorption profile for light incident normally on the emissive surface at $x=0$ (Fig. 2),

$$
G(x)=I_{0} \alpha e^{-\alpha x} .
$$

Here $\alpha(\lambda)$ is the absorption coefficient for light of wavelength $\lambda$. For simplicity, we have neglected photon reflection at both surfaces. Reflection at these surfaces can be incorporated by considering a weighted sum of photon fluxes from both the emissive and non-emissive surfaces of the cathode. ${ }^{9}$

Performing the integral, we arrive at the desired general relation for emission yield incorporating diffusive transport, front and back-surface recombination, and finite size effects,

$$
\begin{aligned}
Y= & \frac{S_{1, \text { emission }}}{\left(S_{1, \text { emission }}+S_{1, \text { recombination }}\right)\left(1+\zeta_{2} e^{-2 d / L}\right)+D / L\left(1-\zeta_{2} e^{-2 d / L}\right)} \\
& \times\left(\frac{\alpha}{\alpha+L^{-1}}\left(1-e^{-\left(\alpha+L^{-1}\right) d}\right)+\frac{\alpha}{\alpha-L^{-1}}\left(1-e^{-\left(\alpha-L^{-1}\right) d}\right) \zeta_{2} e^{-2 d / L}\right) .
\end{aligned}
$$

Equation (7) describes the effects of absorption, emission, and recombination processes on emission yield from a cathode of arbitrary thickness.

For comparison here, we reduce this relation to the simple case of a semi-infinite cathode $(d \rightarrow \infty)$ (compare, e.g., to Ref. 9),

$$
Y_{\text {semi-infinite }}=\frac{S_{1, \text { emission }}}{S_{1, \text { emission }}+S_{1, \text { recombination }}+D / L} \frac{\alpha}{\alpha+L^{-1}}
$$

In both Eq. (7) and the simpler Eq. (8), the emission yield is a product of two terms. The first describes the competition between emission and recombination at the front surface $\left(S_{1 \text {,emission }}+S_{1 \text {,recombination }}\right.$ ), and recombination in the 
bulk (through the term $D / L$, modified in Eq. (7) by the effects of the back surface through $\zeta_{2}$ and cathode thickness $d$ ). The second term relates to the competition between absorption and diffusion in the film of thickness $d$ through their characteristic length scales $\alpha^{-1}$ and $L$, again modified by the effects of finite thickness and the back surface in Eq. (7).

In this work, we use the general yield Eq. (7) to explore the effects of front-surface recombination (Sec. III), backsurface recombination (Sec. IV), and absorption profile (Sec. V) on the total cathode yield. Finally, Sec. VII will examine emission yield using the particular form of $S_{1, \text { emission }}$ for PETE cathodes, which are particularly sensitive to material thickness and surface recombination.

\section{EMISSIVE SURFACE RECOMBINATION}

Of the material parameters relevant for yield, emissivesurface recombination has received the least attention in treatments of traditional photocathodes, due primarily to the high probability of emission at the surface. However, emissive-surface recombination can have a significant impact on photoemission yield in applications with low percollision electron emission probabilities.

To eliminate the complications introduced by the optical absorption profile, we assume that electron generation occurs entirely at the front surface (i.e., $\alpha \rightarrow \infty$ in Eq. (7)). The second term in Eq. (7) then reduces to $\left(1+\zeta_{2} e^{-2 d / L}\right)$, resulting in a simplified yield expression,

$$
Y_{\alpha \rightarrow \infty}=\frac{S_{1, \text { emission }}}{S_{1, \text { emission }}+S_{1, \text { recombination }}+(D / L)\left(1-\zeta_{2} e^{-2 d / L}\right) /\left(1+\zeta_{2} e^{-2 d / L}\right)} .
$$

Equation (9) highlights the competition between emission and recombination at the front surface (first two terms in the denominator) and recombination processes in the bulk and back surface (final term in the denominator). Figure 3 shows the internal quantum yield in Eq. (9) as a function of the front-surface recombination velocity $S_{1, \text { recombination for a }}$ range of front-surface emission velocities $S_{1, \text { emission. }}$ Recombination at the back surface is neglected, and carrier transport properties are similar to those of high-quality p-type GaAs under low injection $\left(D=200 \mathrm{~cm}^{2} / \mathrm{s}, L=10 \mu \mathrm{m}\right.$ and the effective mass of elections in the conduction band is $\left.m_{\mathrm{e}}^{*}=0.063^{*} m_{\mathrm{e}}\right)^{26,27}$

As front-surface recombination increases (from $10^{2} \mathrm{~cm} / \mathrm{s}$ to $10^{7} \mathrm{~cm} / \mathrm{s}$ in Fig. 3), yield correspondingly decreases. However, as seen in Fig. 3, the rate of this decrease depends strongly on both the thickness and the emission probability. While for each curve, a large recombination velocity results in a low yield, reducing surface recombination below a certain threshold value has minimal effect. This crossover point, above which an increase in surface recombination leads to a rapid reduction in yield, is illustrated in Fig. 3 as a dashed grey line. To the left of the dashed line, which intersects the yield curves at $90 \%$ of their maximum value, bulk recombination dominates, and yield is not sensitive to surface recombination. To the right of the dashed line, yield quickly decreases with increasing interface recombination. Optimizing emission for a given application requires moving from the right side of the dashed line to the left.

The crossover point between the two regimes is set by the magnitude of bulk recombination. Between Figs. 3(a) and $3(\mathrm{c})$, bulk recombination $(D / L)\left(1-e^{-2 d / L}\right) /\left(1+e^{-2 d / L}\right)$ increases two orders of magnitude, from $2 \times 10^{3} \mathrm{~cm} / \mathrm{s}$ to $1.5 \times 10^{5} \mathrm{~cm} / \mathrm{s}$, as the film thickness $d$ increases from $100 \mathrm{~nm}$ to $10 \mu \mathrm{m}$. The increase in bulk recombination results in a shift in the crossover point to higher surface recombination velocities, as well as a decrease in the maximum achievable yield. (a) $100 \mathrm{~nm}$

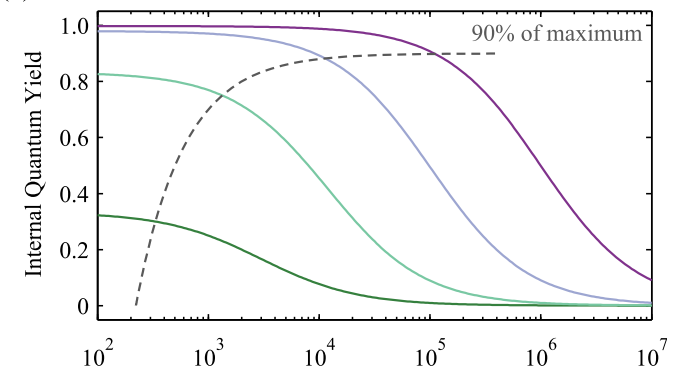

(b) $1 \mu \mathrm{m}$

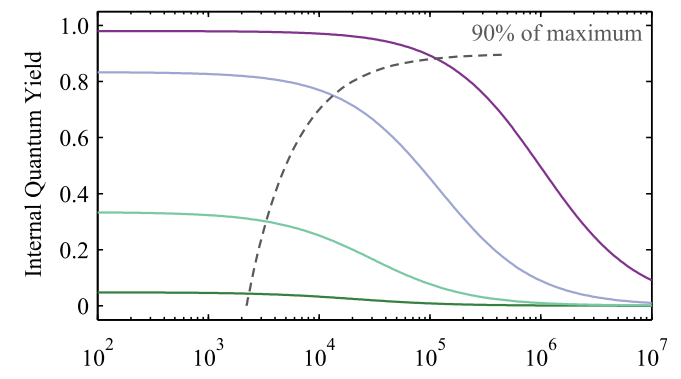

(c) $10 \mu \mathrm{m}$

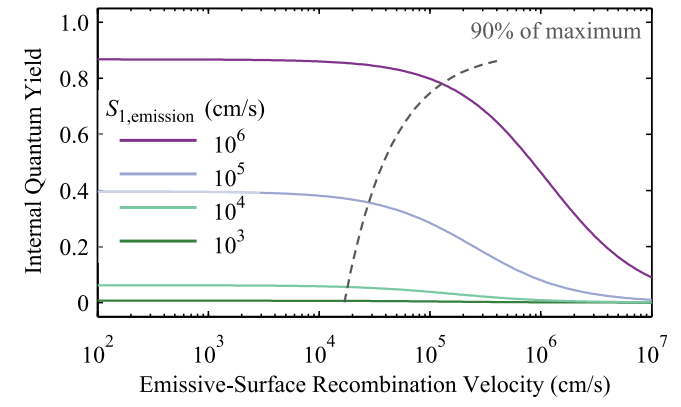

FIG. 3. Effect of front-surface recombination on yield. Internal quantum efficiency is plotted as a function of the front-surface recombination velocity $S_{1, \text { recombination }}$ for a range of emission velocities $S_{1, \text { emission }}$ from $10^{3} \mathrm{~cm} / \mathrm{s}$ to $10^{6} \mathrm{~cm} / \mathrm{s}$. The film thicknesses are (a) $100 \mathrm{~nm}$, (b) $1 \mu \mathrm{m}$, and (c) $10 \mu \mathrm{m}$. Here and in subsequent figures, other parameters are similar to p-type GaAs: electron diffusion length $L=10 \mu \mathrm{m}$, diffusion coefficient $D=200 \mathrm{~cm}^{2} / \mathrm{s}$. 
To achieve practical emission yields in thick cathodes $(d \geq L$, Fig. 3(c)), the high rate of bulk recombination necessitates that the emission velocity $S_{1, \text { emission }}$ be high. For instance, for the high bulk recombination rate in a $10 \mu \mathrm{m}$ thick cathode Fig. 3(c), the maximum possible yields for emission velocities $S_{1, \text { emission }}$ of $10^{3}, 10^{4}, 10^{5}$, and $10^{6} \mathrm{~cm} / \mathrm{s}$ are $0.6 \%, 6 \%, 40 \%$, and $87 \%$, respectively. This strong dependence of yield on emission velocity supports the traditional emphasis on increasing the electron emission probability by minimizing the work function in NEA photocathodes. ${ }^{28,29}$

However, even for cathodes with high emission velocities, emission yield can still be limited if recombination is more likely than emission at the front surface. Indeed, in typical NEA cathodes, emission probabilities ( $B$ in Eq. (1)) are commonly observed to be on the order of $30 \% .^{30}$ This suggests that recombination at the emissive surface is at least 2-3 times more likely than emission there, implying that NEA devices remain to the right of the dashed lines in Fig. 3. This indicates that surface recombination is a critical parameter for both NEA and PETE cathode yield, and a comprehensive theoretical treatment of photocathodes must include an analysis of the impact of surface recombination on device performance. Fig. 3 illustrates that passivation of the emissive surface may noticeably boost emission yields in conventional photocathodes, as surface recombination velocities on the order of $10^{3}-10^{4} \mathrm{~cm} / \mathrm{s}$ are readily achievable in GaAs that has been passivated chemically or by AlGaAs or GaInP. ${ }^{31-33}$ This largely unexplored route to increasing efficiency may be fruitful in practice because commonly used cesium-based surface coatings typically degrade over time in ultra-high vacuum systems, consequently decreasing emission probability and hence device performance. Reducing the surface recombination velocity through passivation would mitigate this decay in emission yield during this degradation process.

\section{BACK SURFACE RECOMBINATION}

The general yield relation (7) deviates from the semiinfinite yield relation (8) primarily through the recurring factor of $\zeta_{2} e^{-2 d / L}$. This factor accounts for recombination at the back surface through the reduced recombination velocity $\zeta_{2}=\left(D / L-S_{2}\right) /\left(D / L+S_{2}\right)$, modified by the probability $e^{-2 d / L}$ that an electron traverses the round-trip distance $2 d$ between the two surfaces. A positive value of $\zeta_{2}$ describes a back surface with low recombination, with $\zeta_{2}=1\left(S_{2}=0\right)$ corresponding to a surface that reflects all incident electrons back into the bulk. A negative value describes a poor quality back surface, with $\zeta_{2}=-1$ $\left(S_{2} \rightarrow \infty\right)$ indicating that every electron incident on the back surface recombines there. When $\zeta_{2}=0\left(S_{2}=D / L\right)$, the probability of recombination at the back surface is identical to the probability in a semi-infinite sample that an electron at a distance $d$ from the front surface diffuses away and recombines deeper in the bulk.

To demonstrate the effect of back-surface recombination on yield, we rewrite Eq. (9) to focus on the reduced recombination velocity $\zeta_{2}$, again assuming that electron generation occurs only at the front surface,

$$
Y_{\alpha \rightarrow \infty}=\frac{S_{1, \text { emission }}\left(1+\zeta_{2} e^{-2 d / L}\right)}{\left(S_{1, \text { emission }}+S_{1, \text { recombination }}\right)\left(1+\zeta_{2} e^{-2 d / L}\right)+D / L\left(1-\zeta_{2} e^{-2 d / L}\right)} .
$$

This form makes the effect of the back surface clear: back-surface recombination weights front-surface emission and recombination $\left(S_{1, \text { emission }}+S_{1 \text {,recombination }}\right)$, and bulk recombination $(D / L)$, by factors of $\left(1 \pm \zeta_{2} e^{-2 d / L}\right)$. As recombination at the back surface decreases ( $\zeta_{2}$ increases), the magnitude of bulk recombination relative to surface processes can decrease significantly.

Fig. 4 displays the influence of back-surface quality on emission yield assuming absorption occurs at the front surface for the same thicknesses and emission velocities as in Fig. 3. Also shown on the top axis are representative values of the reduced recombination velocity $\zeta_{2}$. At $\zeta_{2}=0$ $\left(S_{2}=D / L\right)$, the cathode thickness does not influence the internal yield for absorption near the front surface. As a result, the yield at $\zeta_{2}=0$ is determined exclusively by the emission velocity $S_{1, \text { emission }}$ and is identical between the three panels (a)-(c).

An increase or decrease of $\zeta_{2}$ results in a corresponding increase or decrease of emission yield, and the magnitude of this change is determined by the cathode thickness through $e^{-2 d / L}$. For the thickest cathode shown here (Fig. 4(c)), where the cathode thickness $d$ is equal to the diffusion length $L=10 \mu \mathrm{m}$, yield is relatively insensitive to back-surface recombination: the yield when back-surface recombination approaches zero $\left(S_{2}=0, \zeta_{2}=1\right)$ is at most $30 \%$ larger than its value when back-surface recombination is equal to bulk recombination $\left(\zeta_{2}=0, S_{2}=2 \times 10^{5} \mathrm{~cm} / \mathrm{s}\right)$.

In contrast, for a thin cathode (Fig. 4(a)), back-surface recombination $\zeta_{2}$ strongly influences emission yield, and the recombination at the back surface becomes as important to optimize as recombination at the emissive surface (compare to Fig. 3(a)). This impact is further magnified if the probability of emission is low, as photogenerated carriers remain in the cathode longer and encounter the back surface more frequently. For a thin cathode with thickness $d=100 \mathrm{~nm}$ and front-surface emission/recombination velocity $S_{1 \text {,emission }}$ $=10^{3} \mathrm{~cm} / \mathrm{s}$ in Fig. 4(a), the yield for an electron generated near the surface increases by a factor of $\sim 50$ as the backsurface recombination decreases from the bulk value of $2 \times 10^{5} \mathrm{~cm} / \mathrm{s}$ to zero. This example highlights the importance 
(a) $100 \mathrm{~nm} \quad \zeta_{2}=0.99 \quad 0.9 \quad 0.5 \quad 0 \quad-0.5 \quad 0.9$

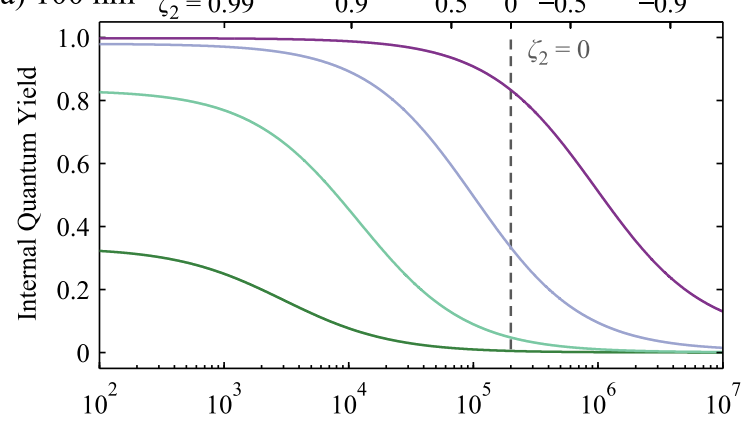

(b) $1 \mu \mathrm{m}$

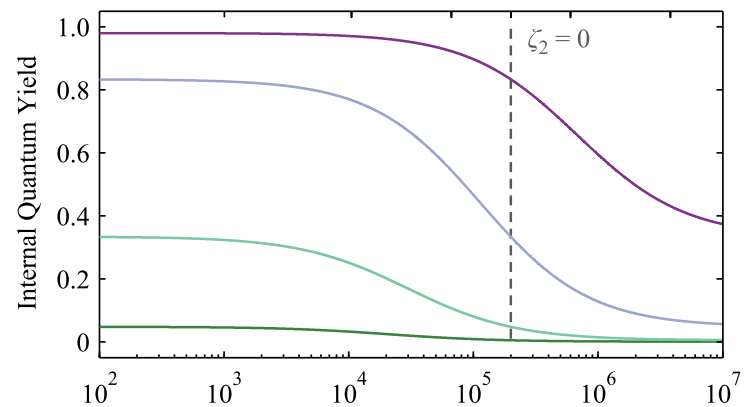

(c) $10 \mu \mathrm{m}$

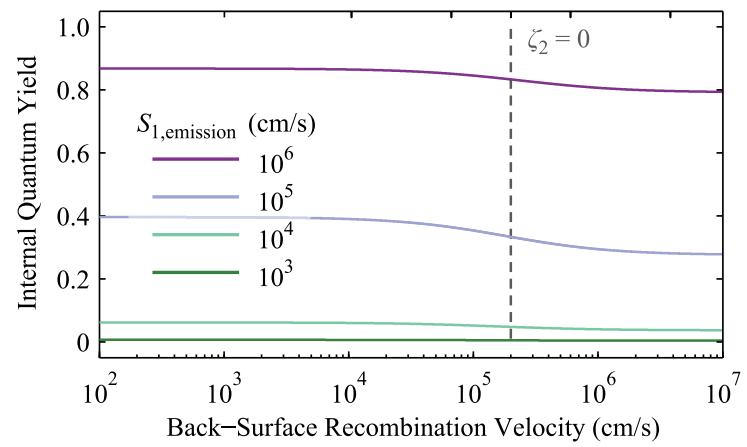

FIG. 4. Effect of back-surface recombination on yield. Internal quantum efficiency is plotted as a function of the back-surface recombination velocity $S_{2}$ for a range of emission velocities $S_{1 \text {,emission }}$ from $10^{3} \mathrm{~cm} / \mathrm{s}$ to $10^{6} \mathrm{~cm} / \mathrm{s}$. Representative values of $\zeta_{2}=\left(D / L-S_{2}\right) /\left(D / L+S_{2}\right)$ are shown on the upper axis, and a dashed line is shown at $\zeta_{2}=0$. The film thicknesses are (a) $100 \mathrm{~nm}$, (b) $1 \mu \mathrm{m}$, and (c) $10 \mu \mathrm{m}$. For simplicity, recombination at the front surface is neglected $\left(S_{1, \text { recombination }}=0\right)$.

of engineering the thickness of cathodes based on PEA materials, as a conduction-band electron may need to encounter the front surface numerous times before gaining sufficient thermal energy to escape into vacuum.

\section{ABSORPTION}

Up to this point, we have neglected absorption to focus on recombination at the surfaces. We now consider the effects of total photon absorption and the initial distribution of carriers in Eq. (6) to complete the description of emission efficiency in finite-thickness photoemitters. The restrictive assumption on photon collection used here, in which incident photons can pass through the cathode only once before they are lost, is by no means required in real devices, and the efficiency of thin photocathodes can be improved considerably using a reflective back surface and light-trapping techniques. $^{34-37}$

Nonetheless, this simplified absorption model illustrates the competition between the length scale for absorption $\alpha^{-1}$ and the characteristic lengths for electron transport. In a semi-infinite cathode, the relevant transport scale is simply the diffusion length $L$, and the absorption profile then affects yield through the simple factor $\alpha /\left(\alpha+L^{-1}\right)$ (Eqs. (1) and (8)). However, in a cathode of finite thickness $d$, two additional effects become important: incomplete photon absorption, and reflection and recombination of carriers excited close to the back surface. These effects contribute to the yield (Eq. (7)) through a sum of two terms,

$\frac{\alpha}{\alpha+L^{-1}}\left(1-e^{-\left(\alpha+L^{-1}\right) d}\right)+\frac{\alpha}{\alpha-L^{-1}}\left(1-e^{-\left(\alpha-L^{-1}\right) d}\right) \zeta_{2} e^{-2 d / L}$.

The first term accounts for incomplete light capture in a film of thickness $d$. This can be seen by setting $S_{1, \text { emission }}+$ $S_{1 \text {,recombination }} \gg D / L$ and $S_{2}=D / L\left(\zeta_{2}=0\right)$ in Eq. (7) to eliminate the competition between surface and bulk recombination at both surfaces and focus solely on absorption. The resulting expression, $\frac{\alpha}{\alpha+L^{-1}}\left(1-e^{-\left(\alpha+L^{-1}\right) d}\right)$, is precisely the first term in Eq. (11).

The second term accounts for additional reflection or recombination at the back surface. To illustrate this, we exchange $x_{0} \leftrightarrow\left(d-x_{0}\right)$ in $G\left(x_{0}\right)$ (Eq. (6)) to derive an equation for yield for a cathode in transmission mode, in which photons are incident on the back surface,

$$
\begin{aligned}
Y_{\text {transmission }}= & \frac{S_{1, \text { emission }}}{\left(S_{1, \text { emission }}+S_{1, \text { recombination }}\right)\left(1+\zeta_{2} e^{-2 d / L}\right)+D / L\left(1-\zeta_{2} e^{-2 d / L}\right)} \\
& \times\left(\frac{\alpha}{\alpha-L^{-1}}\left(1-e^{-\left(\alpha-L^{-1}\right) d}\right)+\frac{\alpha}{\alpha+L^{-1}}\left(1-e^{-\left(\alpha+L^{-1}\right) d}\right) \zeta_{2}\right) e^{-d / L} .
\end{aligned}
$$

In practice, this transmission mode formula is also useful for reflection mode cathodes, as it describes the yield due to photons which reflect at the back surface. Once again, setting $S_{1, \text { emission }}+S_{1 \text {,recombination }} \gg D / L$ and $S_{2}=D / L$ in Eq. (12) to focus solely on absorption yields a result
$\frac{\alpha}{\alpha-L^{-1}}\left(1-e^{-\left(\alpha-L^{-1}\right) d}\right) e^{-d / L}$ that is identical to the second term in Eq. (11), except for one factor of $e^{-d / L}$ relating to transport between the two surfaces. Thus, the first absorption term describes emission as if electrons could diffuse through the back surface, while the second term is directly related to 
the "yield" of electrons that encounter (and then possibly reflect from) the back surface.

Figure 5 demonstrates the influence of the absorption profile by showing yield versus thickness for an absorption coefficient $\alpha=10^{4} \mathrm{~cm}^{-1}$ similar to the value near the GaAs band edge. ${ }^{38,39}$ In each panel of Fig. 5, the black curve illustrates the effect of absorption without considering the effects of back-surface recombination $\left(\zeta_{2}=0, S_{2}=D / L\right)$. In reflection mode (left column), the black curves increase as a larger fraction of photons are absorbed. In transmission mode (right column), the yields eventually decrease as electrons excited close to the back surface of a thick cathode cannot easily diffuse to the emissive surface before they recombine. In either reflection or transmission mode, the yield is close to its maximum after a few absorption lengths $\left(\alpha^{-1}\right)$ : $\sim 1-3 \mu \mathrm{m}$ (compare, e.g., to Ref. 9). This thickness is somewhat reduced in cathodes with an optically reflective back surface, as photons would have additional passes through the cathode.

The influence of the back surface is shown in the spread from purple $\left(S_{2} \rightarrow \infty\right.$, very high recombination) to green $\left(S_{2} \rightarrow 0\right.$, very low recombination) in Fig. 5. As seen in Sec. $\mathrm{IV}$, the influence of the back surface is greatest when the cathode is thin. At the optimal thickness for light absorption here of $1-3 \mu \mathrm{m}$, the quality of the back surface has a reduced influence on yield, particularly for cathodes in reflection mode with high emission probabilities. For instance, in Fig. 5(a), in which $S_{1, \text { emission }}=10^{6} \mathrm{~cm} / \mathrm{s}$, the yield at the optimal thickness of around $3 \mu \mathrm{m}$ is only $90 \%$ greater for no back-surface recombination (i.e., $S_{2}=0$ ) than for infinite back-surface recombination $\left(S_{2} \rightarrow \infty\right)$. This relatively minor impact on performance explains why research on NEA

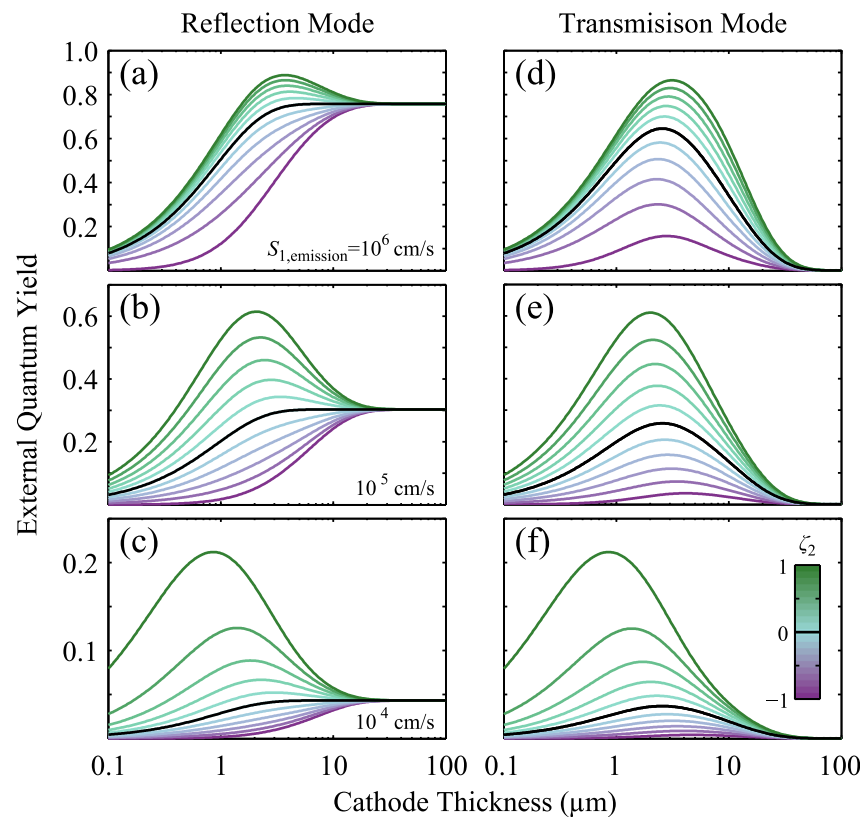

FIG. 5. Effect of cathode thickness on yield for an absorption coefficient $\alpha=10^{4} \mathrm{~cm}^{-1}$. In the left column (a)-(c), the cathode is in reflection mode, and electrons are emitted from the illuminated surface. In the right column (d)-(f), the cathode is in transmission mode, and electrons are emitted from the non-illuminated surface. In the first row $(a, d)$, the emission/recombination velocity is $S_{1, \text { emission }}=10^{6} \mathrm{~cm} / \mathrm{s}$, while in the second (b,e) and third rows $(\mathrm{c}, \mathrm{f})$, the velocities are $10^{5} \mathrm{~cm} / \mathrm{s}$ and $10^{4} \mathrm{~cm} / \mathrm{s}$, respectively. As in Fig. $4, L=10 \mu \mathrm{m}, D=200 \mathrm{~cm}^{2} / \mathrm{s}$, and $S_{1, \text { recombination }}=0$. cathodes has more commonly focused on back-surface quality in the context of transmission-mode devices. ${ }^{9,17}$

In transmission-mode geometry, recombination at the back surface is typically more important than in reflection mode, since the cathode must be thin to ensure efficient transport to the emissive surface. Back-surface quality is most important when the probability of emission is minimal, as electrons may encounter each surface multiple times before leaving the conduction band through either recombination or emission. When the emission velocity is at a minimum of $10^{4} \mathrm{~cm} / \mathrm{s}$ (shown in Fig. 5(f)), decreasing the backsurface recombination velocity from the bulk value of $2 \times$ $10^{5} \mathrm{~cm} / \mathrm{s}$ (central black trace) to $0 \mathrm{~cm} / \mathrm{s}$ (highest green trace) increases the total emission yield by nearly an order of magnitude from $3.7 \%$ to $21 \%$. Nonetheless, even here, the recombination velocity at the back surface is less important for emission yield than the emission velocity at the emissive surface: comparing the black traces in Figs. 5(e) and 5(f), we see that increasing the front surface emission velocity by a single order of magnitude from $10^{4} \mathrm{~cm} / \mathrm{s}$ to $10^{5} \mathrm{~cm} / \mathrm{s}$ increases the overall emission yield to an even larger degree, from $3.7 \%$ to nearly $26 \%$.

\section{EXTENSION TO PHOTON-ENHANCED THERMIONIC EMISSION CATHODES}

Photon-enhanced thermionic emission (PETE) is a recently proposed electron emission mechanism that is promising for solar energy conversion and photocathode applications. $^{12}$ In a PETE cathode, photons incident on a semiconductor film excite electrons from the valence band to the conduction band. These electrons thermalize and diffuse in the film. At the film surface, carriers encounter a positive electron affinity barrier, and only electrons with sufficient thermal energy can overcome this barrier and escape into vacuum. Any increase in this electron affinity raises the output voltage of the PETE device, but increased electron affinity also results in a reduction in the emission velocity, and hence current, potentially reducing the device's power conversion efficiency. This section explores in detail the implications of the yield Eq. (7) for PETE.

In a PETE cathode, the emission velocity $S_{1, \text { emission }}$ can be defined as ${ }^{12}$

$$
S_{1, \mathrm{emission}}=\left(1-R_{\mathrm{e}}\right)\left\langle v_{x}\right\rangle e^{-\chi / k T},
$$

where the factor $e^{-\chi / k T}$ represents the probability that an electron reaching the surface has sufficient thermal energy $\sim k T$ to overcome the electron affinity barrier $\chi$ and escape into vacuum. In Eq. (13), $\left\langle v_{x}\right\rangle=\sqrt{k T / 2 \pi m^{*}}$ is the average magnitude of an electron's thermal velocity perpendicular to the surface. We assume that the conduction band is parabolic and isotropic with effective mass $m^{*}$ and carriers are completely thermalized at the lattice temperature $T$. For simplicity, we furthermore assume that carriers do not reflect at the surface, $R_{\mathrm{e}}=0$.

The dependence of internal emission yield on temperature shown in Fig. 6 illustrates the competition between front-surface recombination and emission from a PETE 
(a) semi-infinite

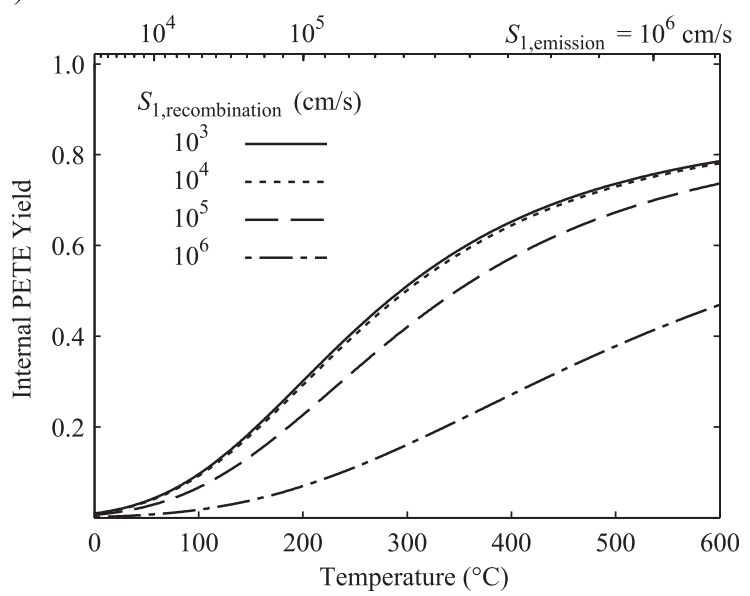

(b) $100 \mathrm{~nm}$

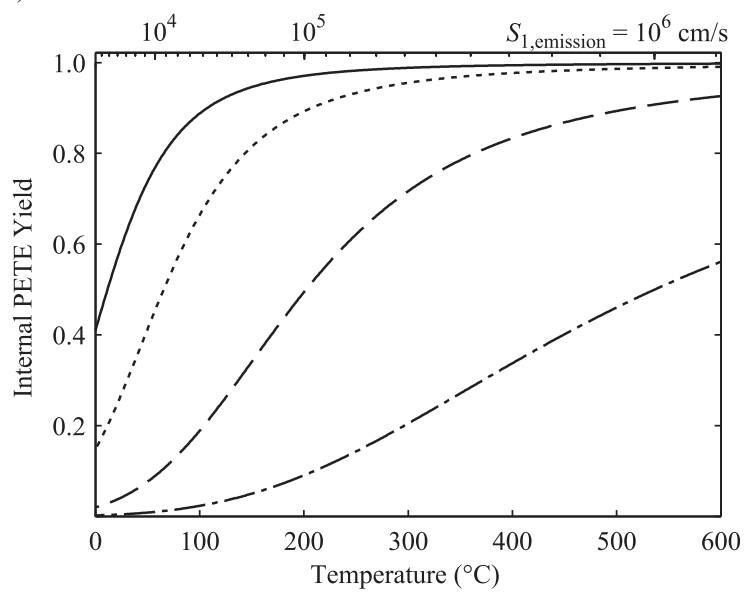

FIG. 6. Dependence of internal emission yield on temperature for a cathode with electron affinity $\chi=0.2 \mathrm{eV}$. In (a), the cathode is semi-infinite, and in (b), its thickness is $100 \mathrm{~nm}$. The four traces represent different values of the front-surface recombination velocity $S_{1 \text {,recombination }}$ ranging from $10^{3} \mathrm{~cm} / \mathrm{s}$ to $10^{6} \mathrm{~cm} / \mathrm{s}$. The effective mass of electrons in the conduction band is 0.063 of the electron mass in vacuum, and to focus on PETE, we ignore the temperature dependence of the effective mass, $D, L, \alpha$, and $S_{1, \text { recombination }}$.

cathode with electron affinity $\chi=0.2 \mathrm{eV}$. In Fig. 6(a), the cathode is semi-infinite in extent, and Eq. (7) takes the simple form (compare to Eq. (8)),

$Y_{\text {PETE,bulk }}=\left(\frac{\left\langle v_{x}\right\rangle e^{-\chi / k T}}{\left\langle v_{x}\right\rangle e^{-\chi / k T}+S_{1, \text { recombination }}+D / L}\right)\left(\frac{\alpha}{\alpha+L^{-1}}\right)$.

In a semi-infinite cathode, surface recombination does not affect yield strongly until its value approaches the relatively large bulk recombination velocity $D / L=2 \times 10^{5} \mathrm{~cm} / \mathrm{s}$. As a result, recombination in the bulk is dominant in the first three traces of Fig. 6(a) $\left(S_{1, \text { recombination }}=10^{3} \mathrm{~cm} / \mathrm{s}\right.$ to $\left.10^{5} \mathrm{~cm} / \mathrm{s}\right)$, and emission only becomes efficient around $300{ }^{\circ} \mathrm{C}$, when the

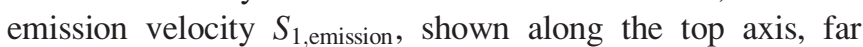
exceeds $10^{5} \mathrm{~cm} / \mathrm{s}$.

Bare p-type GaAs, however, is known to have a high surface recombination velocity on the order of or greater than $S_{1, \text { recombination }}=10^{6} \mathrm{~cm} / \mathrm{s}^{20,40,41}$ a level that would effectively quench photon-enhanced thermionic emission. In Fig. 6(a), the theoretical yield for $S_{1 \text {,recombination }}=10^{6} \mathrm{~cm} / \mathrm{s}$ at the high temperature of $600{ }^{\circ} \mathrm{C}$ is less than $50 \%$. High recombination near the emissive surface would similarly explain the low quantum efficiencies seen in proofof-concept measurements of PETE on GaN. ${ }^{12}$ In contrast, higher yields are achievable in Fig. 6(a) below $300^{\circ} \mathrm{C}$ for surface recombination velocities of $10^{3}-10^{4} \mathrm{~cm} / \mathrm{s}$.

The competition between emission and recombination at the emissive surface is more critical in a thin cathode, where bulk recombination is reduced. Fig. 6(b) repeats the calculations of Fig. 6(a) for a $100 \mathrm{~nm}$ GaAs film with negligible back-surface recombination. The yield for this thickness is approximately given by

$$
Y_{\mathrm{PETE}, \mathrm{film}}=\frac{\left\langle v_{x}\right\rangle e^{-\chi / k T}}{\left\langle v_{x}\right\rangle e^{-\chi / k T}+S_{1, \text { recombination }}+d / \tau}\left(1-e^{-\alpha d}\right) .
$$

Here the bulk recombination rate is reduced from $D / L$ to $d / \tau$, where $\tau$ is the electron lifetime. As seen in Fig. 6(b), emission from a thin film is more efficient and even more sensitive to front-surface recombination than emission from a semi-infinite cathode. If $S_{1 \text {,recombination }}=10^{3} \mathrm{~cm} / \mathrm{s}$, internal emission yield is nearly saturated at $200{ }^{\circ} \mathrm{C}$. However, the external yield under these conditions is at most around $10 \%$ due to incomplete light absorption for $\alpha=10^{4} \mathrm{~cm}^{-1}$, considerably smaller than in the bulk case. This restriction emphasizes the need for effective light-trapping techniques to boost absorption in a thin-film PETE cathode. ${ }^{35-37,42}$

To maximize the power conversion efficiency of a PETE converter, however, it is not sufficient to simply maximize the emission yield by adjusting the device thickness and surface recombination rates. Optimizing electron affinity is also critical for PETE conversion efficiency, as an increase in electron affinity directly increases the output voltage. However, in some temperature ranges, an increase in electron affinity may result in an unacceptable reduction in emission current. For instance, below $\sim 100{ }^{\circ} \mathrm{C}$ in Fig. 6 (b) for $S_{1, \text { recombination }}=10^{3} \mathrm{~cm} / \mathrm{s}$, a small increase in barrier height from $0.2 \mathrm{eV}$ would significantly decrease emission current. In contrast, if the operating temperature is high enough to saturate the emission current $\left(>200^{\circ} \mathrm{C}\right)$, the same change in electron affinity would have little impact on the emission current. Therefore, to optimize the performance of a PETE device, it is critical to identify the maximum electron affinity at which a target yield is achievable.

Figure 7 plots the electron affinity corresponding to an internal yield of $80 \%$ as a function of the front-surface recombination velocity. The power output is typically close to its maximum around $80 \%$ internal yield in an idealized PETE converter, ${ }^{12}$ and this yield is also attainable in a semiinfinite GaAs cathode, where the probability of emission is limited to at most $\alpha /\left(\alpha+L^{-1}\right) \approx 90 \%$ near the band edge.

As noted previously, bulk recombination is substantial in thick films, and in the semi-infinite cathode shown in Fig. 7(a), it limits yield for front-surface recombination velocities less than $D / L=2 \times 10^{5} \mathrm{~cm} / \mathrm{s}$. As a result, an internal yield exceeding $80 \%$ is barely achievable with an electron affinity near $0.2 \mathrm{eV}$, even at temperatures as high as $600^{\circ} \mathrm{C}$.

In Fig. 7(b), the cathode is $100 \mathrm{~nm}$ thick with negligible back-surface recombination, which reduces the bulk-related 
(a) Semi-infinite

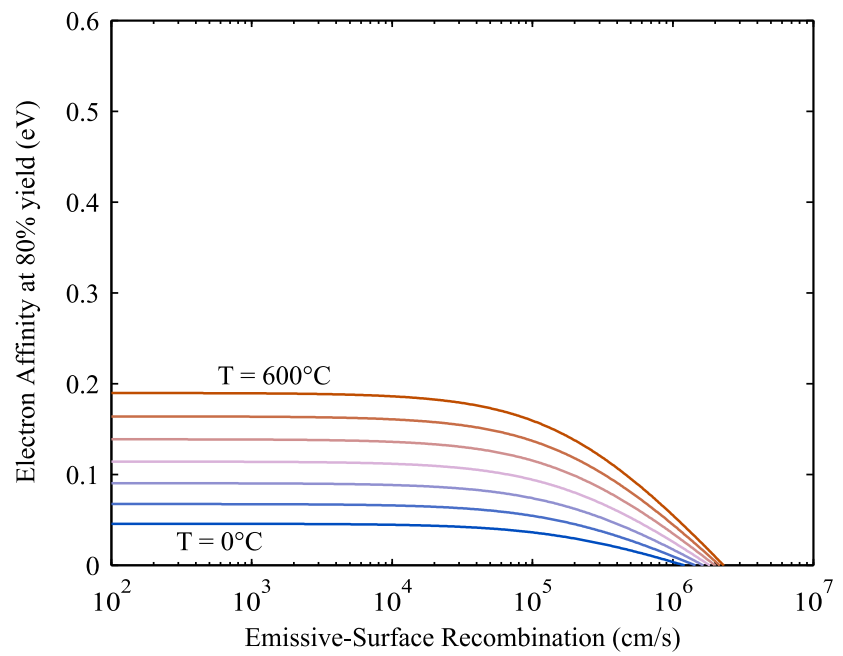

(b) $100 \mathrm{~nm}$

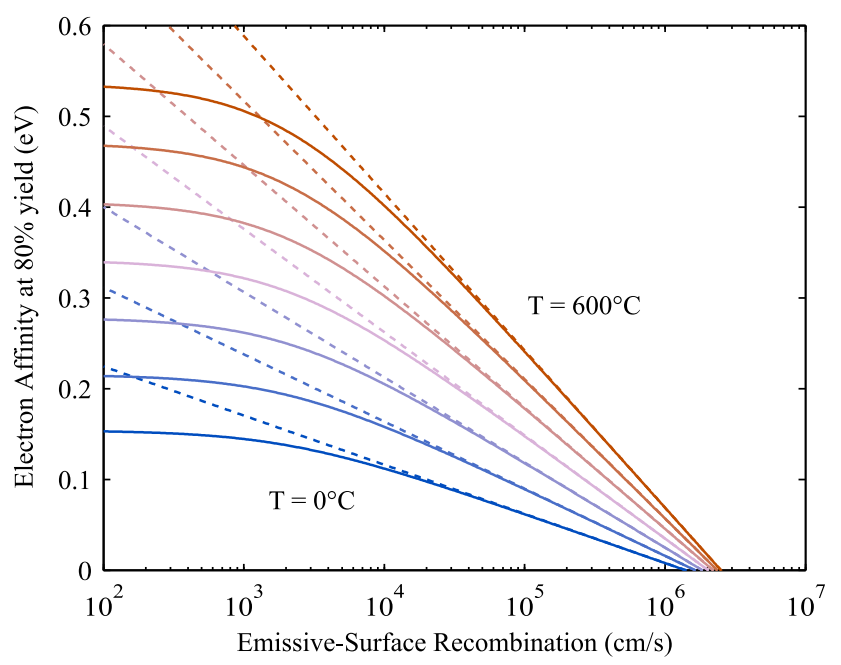

FIG. 7. Electron affinity possible while achieving $80 \%$ internal quantum efficiency as a function of front-surface recombination velocity $S_{1, \text { recombination }}$ for (a) a semi-infinite sample and (b) a $100 \mathrm{~nm}$ thin film, for a range of temperatures from $0^{\circ} \mathrm{C}$ (blue) to $600{ }^{\circ} \mathrm{C}$ (red). Dotted lines indicate the electron affinity possible ignoring recombination in the bulk.

recombination term $(D / L)\left(1-\zeta_{2} e^{-d / L}\right) /\left(1+\zeta_{2} e^{-d / L}\right)$ by two orders of magnitude to $d / \tau=2 \times 10^{3} \mathrm{~cm} / \mathrm{s}$. At this thickness, a device could operate at a higher output voltage because the maximum electron affinity at $80 \%$ yield is over $0.5 \mathrm{eV}$ at $600^{\circ} \mathrm{C}$, significantly higher than in Fig. 7(a). Even larger electron affinities would be possible with improved light trapping and at higher temperatures.

However, for surface recombination velocities exceeding the bulk value of $2 \times 10^{5} \mathrm{~cm} / \mathrm{s}$, high yield is unattainable even with very small barriers, and only NEA cathodes can emit electrons efficiently. This again highlights the need for surface passivation to achieve high PETE efficiencies. Below $S_{1, \text { recombination }}=10^{3}-10^{4} \mathrm{~cm} / \mathrm{s}$, bulk recombination again begins to dominate surface recombination, as seen in Fig. 7(b) by comparing the solid traces to the dashed traces, which show the maximum possible electron affinity assuming that the only competing process to emission is frontsurface recombination. As noted previously, recombination velocities of this order are achievable through common passivation techniques, mitigating the impact of surface recombination on the power output of a thin-film PETE device.

\section{CONCLUSION}

We have presented an analytical model for electron emission from a semiconductor film of arbitrary thickness. The resulting expression for emission yield incorporates recombination velocities at the front and back surfaces as well as bulk transport properties of the film. The emission probability has been examined in both reflection and transmission mode operation, and we have analyzed the implications of this simple expression for modeling devices based on both NEA photoemission and PETE as a physical realization.

We have quantified the advantages of thin films for maximizing internal emission yield from PETE cathodes. The high frequency of surface collisions achieved by reducing material thickness allows electrons to overcome larger electron affinities, increasing the overall power conversion efficiency of a PETE device. However, reducing the thickness also decreases light absorption, highlighting the need for nanostructuring and light-trapping techniques to maximize absorption in thin-film cathodes.

Surface recombination is critical to emission yield due to the low probability of electron emission at the surface. We have shown that the surface recombination velocities required for bulk-dominated recombination, even in thin films, are achievable with chemical surface passivation or at heterostructure interfaces, and we have furthermore proposed surface passivation as a means to increase emission yield from NEA photocathodes.

\section{ACKNOWLEDGMENTS}

The authors would like to thank D. C. Riley and V. K. Narasimhan for guidance and useful discussions. This research was supported by the Global Climate and Energy Project and by the Gordon and Betty Moore Foundation.

${ }^{1}$ P. Krizan, IEEE Trans. Nucl. Sci. 48, 941 (2001).

${ }^{2}$ T. Gys, C. D'Ambrosio, H. Leutz, D. Piedigrossi, and D. Puertolas, Nucl. Instrum. Methods Phys. Res. A 355, 386 (1995).

${ }^{3}$ K. L. Jensen, B. L. Jensen, E. J. Montgomery, D. W. Feldman, P. G. O'Shea, and N. Moody, J. Appl. Phys. 104, 044907 (2008).

${ }^{4}$ S. W. Downey, L. A. Builta, D. C. Moir, T. J. Ringler, and J. D. Saunders, Appl. Phys. Lett. 49, 911 (1986).

${ }^{5}$ R. Alley, H. Aoyagi, J. Clendenin, J. Frisch, C. Garden, E. Hoyt, R. Kirby, L. Klaisner, A. Kulikov, R. Miller, G. Mulhollan, C. Prescott, P. Sáez, D. Schultz, H. Tang, J. Turner, K. Witte, M. Woods, A. D. Yeremian, and M. Zolotorev, Nucl. Instrum. Methods Phys. Res. A 365, 1 (1995).

${ }^{6}$ S. H. Kong, J. Kinross-Wright, D. C. Nguyen, R. L. Sheffield, and M. E. Weber, Nucl. Instrum. Methods Phys. Res. A 358, 284 (1995).

${ }^{7}$ R. A. La Rue, K. A. Costello, G. A. Davis, J. P. Edgecumbe, and V. W. Aebi, IEEE Trans. Electron Devices 44, 672 (1997).

${ }^{8}$ G. F. Knoll, Radiation Detection and Measurement, 4th ed. (Wiley, Hoboken, New Jersey, 2010).

${ }^{9}$ R. L. Bell, Negative Electron Affinity Devices (Clarendon, Oxford, 1973).

${ }^{10}$ W. E. Spicer, Appl. Phys. 12, 115 (1977). 
${ }^{11}$ F. Machuca, Y. Sun, Z. Liu, K. Ioakeimidi, P. Pianetta, and R.F.W. Pease, J. Vac. Sci. Technol. B 18, 3042 (2000).

${ }^{12}$ J. W. Schwede, I. Bargatin, D. C. Riley, B. E. Hardin, S. J. Rosenthal, Y. Sun, F. Schmitt, P. Pianetta, R. T. Howe, Z.-X. Shen, and N. A. Melosh, Nat. Mater. 9, 762 (2010).

${ }^{13}$ W. E. Spicer, Phys. Rev. 112, 114 (1958).

${ }^{14}$ W. E. Spicer and A. Herrera-Gómez, Proc. SPIE 2022, 18 (1993).

${ }^{15}$ X. H. Wang, B. K. Chang, L. Ren, and P. Gao, Appl. Phys. Lett. 98, 082109 (2011)

${ }^{16}$ Y. J. Zhang, J. J. Zou, J. Niu, J. Zhao, and B. K. Chang, J. Appl. Phys. 110, 063113 (2011)

${ }^{17}$ Y. Z. Liu, J. L. Moll, and W. E. Spicer, Appl. Phys. Lett. 17, 60 (1970).

${ }^{18}$ G. A. Antypas, L. W. James, and J. J. Uebbing, J. Appl. Phys. 41, 2888 (1970).

${ }^{19}$ G. Duggan and G. B. Scott, J. Appl. Phys. 52, 407 (1981).

${ }^{20}$ K. Mettler, Appl. Phys. 12, 75 (1977).

${ }^{21}$ V. V. Afanas'ev and A. Stesmans, J. Appl. Phys. 102, 081301 (2007).

${ }^{22}$ L. W. James and J. L. Moll, Phys. Rev. 183, 740 (1969).

${ }^{23}$ P. Hartmann, J. Bermuth, D. v. Harrach, J. Hoffmann, S. Köbis, E. Reichert, K. Aulenbacher, J. Schuler, and M. Steigerwald, J. Appl. Phys. 86, 2245 (1999).

${ }^{24}$ K. Aulenbacher, J. Schuler, D. v. Harrach, E. Reichert, J. Röthgen, A. Subashev, V. Tioukine, and Y. Yashin, J. Appl. Phys. 92, 7536 (2002).

${ }^{25}$ M. G. Clark, J. Phys. D 9, 2139 (1976).

${ }^{26}$ M. E. Levinshtein, S. L. Rumyantsev, and M. Shur, Handbook Series on Semiconductor Parameters (World Scientific, Singapore, 1996).
${ }^{27}$ J. S. Blakemore, J. Appl. Phys. 53, R123 (1982).

${ }^{28}$ J. S. Escher and G. A. Antypas, Appl. Phys. Lett. 30, 314 (1977).

${ }^{29}$ D. G. Fisher, R. E. Enstrom, J. S. Escher, and B. F. Williams, J. Appl. Phys. 43, 3815 (1972).

${ }^{30}$ J. S. Escher, in Contacts, Junctions, Emitters, Semiconductors and Semimetals, edited by R. K. Willardson and A. C. Beer (Academic, New York, 1981), Vol. 15

${ }^{31}$ S. R. Lunt, G. N. Ryba, P. G. Santangelo, and N. S. Lewis, J. Appl. Phys. 70, 7449 (1991)

${ }^{32}$ R. J. Nelson and R. G. Sobers, Appl. Phys. Lett. 32, 761 (1978).

${ }^{33}$ J. M. Olson, R. K. Ahrenkiel, D. J. Dunlavy, B. Keyes, and A. E. Kibbler, Appl. Phys. Lett. 55, 1208 (1989).

${ }^{34}$ W. D. Gunter, Jr., G. R. Grant, and S. A. Shaw, Appl. Opt. 9, 251 (1970).

${ }^{35}$ E. Yablonovitch, J. Opt. Soc. Am. 72, 899 (1982).

${ }^{36}$ K. Nakayama, K. Tanabe, and H.A. Atwater, Appl. Phys. Lett. 93, 121904 (2008).

${ }^{37}$ Z. Yu, A. Raman, and S. Fan, Proc. Natl. Acad. Sci. USA 107, 17491 (2010).

${ }^{38}$ I. Kudman and T. Seidel, J. Appl. Phys. 33, 771 (1962).

${ }^{39}$ E. D. Palik, Handbook of Optical Constants of Solids (Academic, Orlando, 1985).

${ }^{40}$ M. G. Mauk, S. Xu, D. J. Arent, R. P. Mertens, and G. Borghs, Appl. Phys. Lett. 54, 213 (1989).

${ }^{41}$ J. M. Woodall, G. D. Pettit, T. Chappell, and H. J. Hovel, J. Vac. Sci. Technol. 16, 1389 (1979).

${ }^{42}$ B. G. Lee, P. Stradins, D. L. Young, K. Alberi, T.-K. Chuang, J. G. Couillard, and H. M. Branz, Appl. Phys. Lett. 99, 064101 (2011). 